\title{
Transfer of learning in choice reactions: The roles of stimulus type, response mode, and set-level compatibility
}

\author{
Motonori Yamaguchi • Jing Chen • Robert W. Proctor
}

Published online: 11 March 2015

(C) Psychonomic Society, Inc. 2015

\begin{abstract}
The Simon effect refers to the advantage of responding to spatially compatible stimuli. This effect can be eliminated or even reversed to favor spatially incompatible stimuli after participants practice a choice-reaction task with spatially incompatible mappings (e.g., pressing left and right keys to stimuli on the right and left, respectively). This transfer of incompatible spatial associations has been observed under conditions in which responses were made manually (e.g., keypresses, moving a joystick). The present study used vocal responses to reveal the primary determinants of the transfer effect, dissociating the influences of stimulus type, response mode, and their interaction (set-level compatibility). The results suggest that contextual match between the practice and transfer tasks with respect to stimulus type and response mode determined transfer of incompatible associations to the Simon task, and stimulus type determined the efficiency of acquiring new associations. However, there was little evidence that setlevel compatibility plays any major role in either acquisition or transfer of spatial associations.
\end{abstract}

Keywords Transferoflearning - Spatial representation · Vocal responses $\cdot$ Specificity of learning $\cdot$ Stimulus-response compatibility

\footnotetext{
M. Yamaguchi $(\bowtie)$

Department of Psychology, Edge Hill University,

Ormskirk, Lancashire L39 4QP, UK

e-mail: yamagucm@edgehill.ac.uk

M. Yamaguchi

e-mail: cog.yamaguchi@gmail.com

J. Chen $\cdot$ R. W. Proctor

Department of Psychology, Purdue University, West Lafayette, IN, USA
}

Compatibility between stimulus and response is a major determinant of the efficiency of response selection (Fitts \& Seeger, 1953; Proctor \& Vu, 2006; Welford, 1960). In choicereaction tasks, responses are faster and more accurate when stimuli and responses are spatially compatible than when they are spatially incompatible (e.g., pressing a left key to stimuli on the left, as opposed to pressing the same key to stimuli on the right). This stimulus-response compatibility (SRC) effect occurs when spatial attributes of stimuli are task-relevant or irrelevant (e.g., when responses are determined by the stimulus color rather than the location). When spatial attributes of stimuli are task-irrelevant, the effect is known as the Simon effect (see Lu \& Proctor, 1995, for a review).

The Simon effect has been an important phenomenon of investigation due to its implications about the mechanisms of response selection (Hommel, 2011). For instance, the Simon effect has been a useful tool to investigate the principles underlying transfer of learning (e.g., Baroni, Yamaguchi, Chen, \& Proctor, 2013; Proctor \& Lu, 1999; Tagliabue, Zorzi, Umiltà, \& Bassignani, 2000). In this line of research, participants are first asked to perform a choice-reaction task in which they respond to spatial stimuli (e.g., circles that appear on the left or right of the display) by pressing a key whose location is incompatible with the stimulus location. After performing this incompatible-mapping task, participants transfer to the Simon task, in which they press the left or right key according to non-spatial attributes of stimuli, such as colors. Proctor and $\mathrm{Lu}$ (1999) found that the Simon effect was reversed (i.e., responses were faster when the stimulus and response locations were incompatible) after participants performed three sessions of 600 trials (a total of 1,800 trials) on the incompatible-mapping task. Similarly, Tagliabue et al. (2000) found that the Simon effect was eliminated after performing less than 100 trials on the incompatiblemapping task. These results indicate that participants acquire the incompatible stimulus-response (S-R) associations 
through practice and transfer these incompatible associations to the Simon task even when these associations are no longer relevant to the current task.

This transfer method is useful for identifying factors that affect transfer of learned associations to a novel task. For instance, Tagliabue, Zorzi, and Umiltà (2002) eliminated the Simon effect when the practice task used auditory stimuli and the transfer task used visual stimuli, implying that the transfer effect is not specific to the stimulus modality (also see Proctor, Yamaguchi, \& Vu, 2007; Vu, Proctor, \& Urcuioli, 2003). Proctor, Yamaguchi, Zhang, and Vu (2009) tested three types of spatial stimuli; physical stimulus location (circles that appeared on the left and right), spatial words ("LEFT" and "RIGHT"), and arrows pointing to the left and right, and found that the incompatible mapping transferred to the Simon task when stimulus type matched between the practice and transfer tasks but not when it did not match. For instance, the Simon effect was eliminated when stimuli in the practice and transfer tasks varied in physical locations, whereas the Simon effect was not modulated when stimuli in the practice task were physical locations but those in the transfer task were spatial words. These results indicate that although transfer of learned associations is independent of stimulus modality as long as stimuli convey physical location information (also see $\mathrm{Vu}, 2007)$, it is sensitive to how spatial information is presented.

The specificity of transfer of learning to the learning context is consistent with the idea that knowledge and skill are utilized most effectively when the test context resembles the context in which learning has taken place (Bouton, 1993; Godden \& Baddeley, 1975; Healy, Wohldmann, Sutton, \& Bourne, 2006; Tulving \& Thomson, 1973). However, the previous studies all used manual responses (e.g., pressing keys, deflecting a joystick), and it is not known how much the mode of responding contributes to transfer of learned associations in this paradigm. Therefore, the present study focused on this factor, using vocal responses. By doing so, it allows examining whether the degree of set-level compatibility affects the acquisition and transfer of newly learned associations to a novel task context.

\section{Set-level compatibility for learning and transfer}

Set-level compatibility refers to the compatibility between stimuli and responses at the level of stimulus and response sets, as opposed to individual elements in these sets (Kornblum, Hasbroucq, \& Osman, 1990; Proctor \& Wang, 1997). The degree of set-level compatibility is usually defined in terms of the similarity between stimulus and response sets. For instance, the stimulus set consisting of the words LEFT and RIGHT would be more compatible with the response set consisting of vocal utterance of "left" and "right" than the response set consisting of pressing of left and right keys. A higher degree of set-level compatibility is associated with a larger SRC effect (Kornblum \& Lee, 1995; Wang \& Proctor, 1996) or Simon effect (Baldo, Shimamura, \& Prinzmetal, 1998; Lu \& Proctor, 1994). A higher degree of set-level compatibility, particularly referring to "modality compatibility," is also associated with smaller task-switching cost (Stephan \& Koch, 2010) and dual-task interference (Hazeltine, Ruthruff, $\&$ Remington, 2006). Therefore, set-level compatibility appears to play a significant role in performing cognitive tasks. Nevertheless, less is known about the role of set-level compatibility in learning of new S-R associations (Yamaguchi \& Proctor, 2009).

When responses are made manually, many more practice trials are required to eliminate the Simon effect with spatial words (600 trials) than with physical location stimuli $(<100$ trials; Proctor et al., 2009). These results may reflect the influence of set-level compatibility in the acquisition of new S-R associations; the acquisition may be more efficient when the practice task involves a high degree of set-level compatibility, or it may be due to the type of stimuli; the acquisition of spatial mappings is more efficient with physical location stimuli than spatial words. Also, using a similar transfer method but with the Stroop task (Marini, Iani, Nicoletti, \& Rubichi, 2011), the Stroop effect was reduced after the incompatible-mapping task when the practice and transfer tasks both required manual responses but not when the practice task required manual responses and the transfer task required vocal responses. This outcome is consistent with the finding that contextual match between the practice and transfer tasks with respect to the response mode plays a major role in the transfer of incompatible mappings (Yamaguchi \& Proctor, 2009), but it may also reflect the importance of the type of response itself: the transfer effect may occur only with manual responses. Hence, it is necessary to examine the contribution of the mode of responding to transfer of learned associations to dissociate these possibilities.

\section{Present study}

The present study used the transfer paradigm (Proctor \& Lu, 1999; Tagliabue et al., 2000) to examine the roles of stimulus type, response mode, and set-level compatibility in transfer of incompatible mappings to the Simon task. The main findings in previous studies are that the Simon effect is reduced, or eliminated, after participants practice with the incompatible spatial mappings for less than 100 trials (Tagliabue et al., 2000) and that the modulation is sensitive to the match of stimulus sets between the practice and transfer tasks (Proctor et al., 2009). Also, compared to physical location stimuli (stimuli that appear on the left or right of the display), spatial words ("left" and "right") require more practice trials to modulate the Simon effect (Proctor et al., 2009). As previous 
studies all used manual responses (e.g., pressing keys) that are more compatible with physical location stimuli than with spatial words, it is not clear whether these results reflect the effects of stimulus type alone or the effect of the set-level compatibility between stimuli and response sets.

In the first two experiments, responses were made vocally, and the type of stimuli (physical location vs. spatial words) was varied between the practice and transfer tasks to examine whether set-level compatibility plays a role in the acquisition and transfer of associations. In both experiments, stimuli in the practice task were either physical locations or spatial words, and stimuli in the transfer task were physical locations in Experiment 1 and were spatial words in Experiment 2. These experiments allow dissociating the effect of stimulus type from that of set-level compatibility: If the previous finding, that physical location stimuli modulates the Simon effect with fewer practice trials than spatial words do, is due to the higher degree of set-level compatibility, spatial words should modulate the Simon effect with a small number of practices $(<100$ trials) when responses are made vocally, but physical locations stimuli should not.

In the subsequent two experiments, the mode of responding was manipulated. The design of these experiments was complementary to the design of the first two experiments; stimuli were of the same type between the practice and transfer tasks (physical location stimuli in Experiment 3, and spatial words in Experiment 4), but responses were changed from manual to vocal, or vice versa, between the practice and transfer tasks. Thus, Experiments 3 and 4 dissociated the effect of set-level compatibility from that of the mode of responding.

\section{Experiment 1}

In Experiment 1, participants performed the incompatiblemapping tasks using physical location stimuli (circles that appeared in left and right locations on the display) or spatial words (LEFT and RIGHT that appeared in the center of the display) in the practice task. Participants in both conditions performed the Simon task with the physical location stimuli in the transfer task. Responses were always made by saying "left" or "right" into a microphone. In previous studies using manual keypresses, the Simon effect was reduced when stimuli in the practice task were physical locations but not when they were spatial words if stimuli in the transfer task were physical locations (Proctor et al., 2009). Experiment 1 examined whether these outcomes were due to the degree of setlevel compatibility or due to the type of stimuli. If the match between the two tasks is the primary determinant of the transfer effect, then practice with physical location stimuli should produce a larger modulation of the Simon effect. However, if the higher degree of set-level compatibility is the primary determinant of the transfer effect, practice with spatial words should produce a larger modulation of the Simon effect.

Also, a previous study (Marini et al., 2011) found that practice with a spatially incompatible mapping task can modulate the Stroop effect in the transfer task when using manual keypresses but not when using vocal responses. The lack of the transfer effect could have been due to the altered task context between the practice and transfer tasks (see also Yamaguchi \& Proctor, 2009), but it could also be due to the nature of vocal responses used in the transfer task (e.g., keypress responses are spatially distinct, whereas vocal responses are not. Hence, the present experiment assessed whether the transfer of incompatible mapping can occur when both practice and transfer tasks used vocal responses) as no studies have shown transfer of incompatible mappings with vocal responses.

\section{Method}

Participants Forty-eight undergraduate students at Purdue University participated in the experiment. They received experiment credits toward their introductory psychology courses for participation. All participants reported having normal or corrected-to-normal visual acuity and normal color vision. They were randomly assigned to one of the three groups (see Procedure).

Apparatus and stimuli The apparatus consisted of a 19-in LCD monitor and a personal computer. For the practice task, the stimuli were white circles $(2.5 \mathrm{~cm}$ in diameter $)$ that appeared at the left or right side of the screen $(12.5 \mathrm{~cm}$ from the screen center), or the words LEFT $(1.5 \mathrm{~cm}$ in height, $3 \mathrm{~cm}$ in width) and RIGHT (1.5 cm in height, $4 \mathrm{~cm}$ in width) printed in white that appeared at the center of the screen. For the transfer task, the imperative stimuli were $2.5-\mathrm{cm}$ circles colored in green or red, which appeared on the left or right side of the screen. Responses were collected by speaking into a microphone, saying "left" or "right." The experimenter pressed one of two keys on the keyboard to register participants' responses. Response time (RT) was the interval between onset of a circle and a trigger of voice key.

Procedure The experiment was conducted individually in a dimly lit cubicle. Participants were seated in front of the computer monitor at an unrestricted distance of approximately $60 \mathrm{~cm}$ from the monitor and read on-screen instructions. The experimenter sat in the experiment room in order to register participants' vocal responses.

Participants were assigned randomly to one of the three conditions. In the first condition, participants performed the practice task in which they responded to circles that appeared on the left or right side of the screen (location practice). In the 
second condition, participants performed the practice task in which they responded to the words LEFT and RIGHT (word practice). In the third condition, participants did not perform the practice task (control). For the former two conditions, there was a mandatory break for $5 \mathrm{~min}$ after the practice task. All participants performed the same transfer task in which they responded to colored circles that appeared on the left or right side of the screen.

For the practice session, a trial started with a cross $(0.8 \mathrm{~cm}$ in height and width) presented at the center of the screen, which lasted for $1,000 \mathrm{~ms}$. The cross was replaced by an imperative stimulus. Participants then responded by speaking into the microphone. They said "left" if a circle occurred on the right or the word RIGHT appeared at the center; they said "right" if a circle occurred on the left or the word LEFT appeared. The stimulus stayed on the screen until the microphone detected a vocal response, and a blank display replaced the screen. The experimenter pressed one of two keys to indicate whether participants said "left" or "right" within a 1,000ms time window after the onset of response. If the experimenter failed to register the response within this time window, the trial was recorded as no response. For error or no response, a $400-\mathrm{Hz}$ tone was presented for $500 \mathrm{~ms}$ from the speaker positioned on both sides of the screen. For correct response, a blank screen was presented for $500 \mathrm{~ms}$. The trial ended with another blank screen that lasted for $1,000 \mathrm{~ms}$.

The procedure of the transfer task was essentially the same as that of the practice task, except that the imperative stimuli were color-filled circles that occurred on the left or right of the screen. Participants were encouraged to ignore the circle location and responded to the color by saying "left" and "right." For half the participants in each condition, the "left" and "right" responses were mapped to green and red stimuli, respectively; the mapping was reversed for the other half.

The practice and transfer tasks consisted of 84 and 156 trials, respectively. For both sessions, the first 12 trials were considered warm-up and excluded from the analysis. A session lasted for less than half an hour.

\section{Results}

Trials with RTs of less than $150 \mathrm{~ms}$ or greater than $1,500 \mathrm{~ms}$ were discarded (3.30\% for the practice task; $2.03 \%$ of all trials for the transfer task). For each participant, mean RTs for correct responses and percentage errors (PEs) were computed. Figure 1 depicts RTs in the transfer task. Table 1 summarizes RTs and PEs in the practice task, and Table 2 summarizes PEs in the transfer task. RTs in the transfer task are shown in Fig. 1.

For the practice task, RT was shorter for the location practice group $(M=539 \mathrm{~ms})$ than for the word practice group $(M=$ $671 \mathrm{~ms}), t(30)=4.15, S E=31.67, p<.001$, but PE was not significantly different, $t(30)<0.2, S E=.73$ (see Table 1).

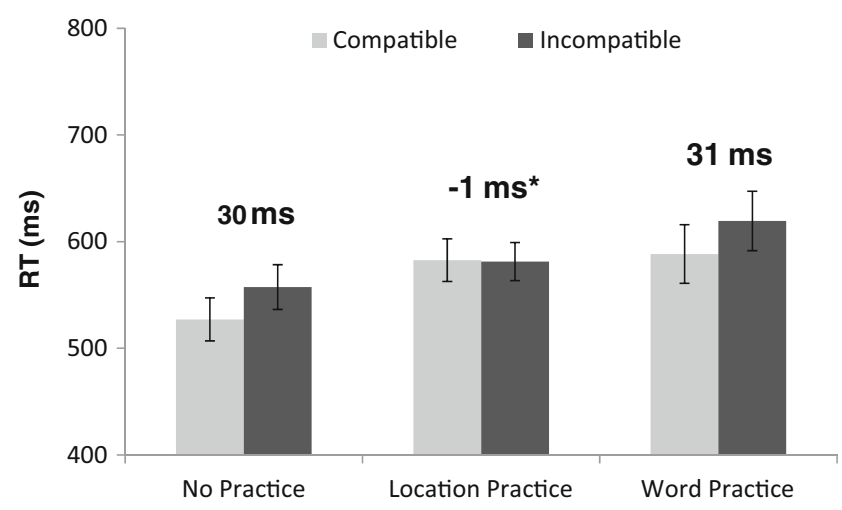

Fig. 1 Response time (RT) as a function of Practice Mode (control, physical location, spatial word) in Experiment 1; the numbers are the Simon effects and an asterisk indicates a significant reduction of the Simon effect compared to the No Practice condition; error bars are standard errors of the means

For the transfer task, RT and PE were submitted to separate analyses of variance (ANOVAs) as a function of Practice Mode (location vs. word vs. control; between-subject) and Compatibility (compatible vs. incompatible; within-subject). For RT, the main effect of Compatibility was significant, $F(1$, $45)=41.77, M S E=230, p<.001, \eta_{\mathrm{p}}^{2}=.481$, yielding a 20 $\mathrm{ms}$ Simon effect $(M \mathrm{~s}=566 \mathrm{~ms}$ and $586 \mathrm{~ms}$ for compatible and incompatible trials, respectively). Compatibility interacted with Practice Mode, $F(2,45)=11.89, M S E=223, p<.001$, $\eta_{\mathrm{p}}{ }^{2}=.346$. The Simon effect was $31 \mathrm{~ms}$ for the control group and $30 \mathrm{~ms}$ for the word practice group, compared to $-1 \mathrm{~ms}$ for the location practice group. There was no main effect of Practice Mode, $F(2,45)=1.92, M S E=16,301, p=.159, \eta_{\mathrm{p}}{ }^{2}=$ .078. For PE, there was no significant effect, $F_{\mathrm{S}}<1.9, p \mathrm{~s}>.1{ }^{1}$

\section{Discussion}

The Simon effect was modulated when stimuli in the practice task were physical locations but not when they were spatial words. The results indicate the importance of contextual match between the practice and transfer tasks in terms of stimulus type, providing little evidence suggesting the importance of set-level compatibility. The modulation of the Simon effect also indicates that incompatible spatial associations do transfer to the Simon task even when responses are not spatially distinct (cf. Marini et al., 2011). Therefore, the present results reinforce the idea that newly acquired spatial associations are utilized more effectively when contextual features present during the acquisition are also present at test (Yamaguchi \& Proctor, 2009). Nevertheless, it is still possible that incompatible associations were acquired quickly with spatial words due to the higher degree of set-level compatibility but were not expressed in the transfer task due to the altered task context. Thus, Experiment 2 tested the same manipulations in the practice task but with spatial words in the transfer task. 
Table 1 Response time (RT) and percentage errors (PE) in the practice tasks of Experiments 1-4

\begin{tabular}{|c|c|c|c|c|c|}
\hline & Practice stimulus/response & Transfer stimulus/response & Number of practice trials & RT & $\mathrm{PE}$ \\
\hline \multirow[t]{2}{*}{ Experiment 1} & Location/Vocal & Location/Vocal & 84 & 539 & 1.82 \\
\hline & Word/Vocal & Location/Vocal & 84 & 671 & 1.68 \\
\hline \multirow[t]{4}{*}{ Experiment 2} & Location/Vocal & Word/Vocal & 84 & 566 & 2.06 \\
\hline & Word/Vocal & Word/Vocal & 84 & 624 & 2.14 \\
\hline & Location/Vocal & Word/Vocal & 168 & 543 & 2.02 \\
\hline & Word/Vocal & Word/Vocal & 168 & 638 & 1.70 \\
\hline \multirow[t]{2}{*}{ Experiment 3} & Location/Vocal & Location/Keypress & 84 & 475 & 1.66 \\
\hline & Location/Keypress & Location/Vocal & 84 & 458 & 2.09 \\
\hline \multirow[t]{2}{*}{ Experiment 4} & Word/Vocal & Word/Keypress & 168 & 612 & 2.03 \\
\hline & Word/Keypress & Word/Vocal & 168 & 586 & 3.85 \\
\hline
\end{tabular}

\section{Experiment 2}

Experiment 2 examined transfer of incompatible spatial mapping to the Simon task using spatial words with vocal responses. Stimuli in the practice task were physical location stimuli or spatial words. Set-level compatibility was higher for the transfer task of the present experiment than that of Experiment 1, and there was a contextual match between the practice and transfer tasks when spatial words were used in the practice task as well. Thus, if set-level compatibility plays a major role, practice with spatial words should produce a larger modulation of the Simon effect than practice with physical location stimuli.

The present experiment also examined whether the degree of set-level compatibility allowed more efficient acquisition of incompatible associations. With manual responses, practice with spatial words required more trials to yield the transfer effect than did practice with physical location stimuli (Proctor et al., 2009). If this outcome was due to high set-level compatibility between physical locations and manual responses, practice with spatial words would require a small number of practice trials $(<100$ trials $)$ to modulate the Simon effect. If learning depends on stimulus type, a larger number of practice trials would be required for spatial words than for physical locations even when responses are made vocally. Thus, the present experiment also manipulated the number of practice trials across groups.

\section{Method}

Participants Eighty undergraduate students at Purdue University participated in the present study. They were divided into five groups of 16 participants (see Procedure). All participants received experimental course credits toward their psychology

\footnotetext{
${ }^{1}$ As shown in Table 2, PE was larger for compatible trials than for incompatible trials, reversing the direction of the Simon effect, but this effect was not statistically significant, $t(15)=1.38, p=.19$.
}

courses, and they reported having normal or corrected-tonormal visual acuity and normal color vision. None had participated in Experiment 1.

Apparatus, Stimuli, and Procedure Experiment 2 followed the procedure of Experiment 1, with the following changes: Two groups of participants were assigned to the conditions in which there were 84 practice trials with spatial words or physical locations, and another two groups were assigned to the conditions in which there were 168 practice trials with spatial words or physical locations. The final group had no practice trials. The stimuli were identical with those used in Experiment 1. All groups performed the same Simon task with spatial words, the words LEFT and RIGHT colored in green or red. Half the participants in each group said "left" to green words and "right" to red words, and the other half said "right" to green words and "left" to red words. Experiment 2 was essentially the same as Experiment 1 in other respects.

\section{Results}

The percentages of discarded trials were $2.27 \%$ for the practice task and $2.21 \%$ for the transfer task, with the same filtering criteria as in Experiment 1. RTs and PEs for the practice task are summarized in Table 1, and RTs and PEs for the transfer task are given in Fig. 2 and Table 2, respectively.

RTs and PEs for the practice task were submitted to separate ANOVAs as a function of Practice Mode (physical location vs. spatial word) and Practice Length (84 vs. 168). Both factors were between-subject variables. For RT, there was a significant main effect of Practice Mode, $F(1,60)=10.63$, $M S E=8781, p=.002, \eta_{\mathrm{p}}{ }^{2}=.150$, reflecting shorter RT for physical locations $(M=555 \mathrm{~ms})$ than for spatial words $(M=$ $631 \mathrm{~ms})$. No other effects were significant, $F \mathrm{~s}<1$. For PE, no effects were significant, $F_{\mathrm{S}}<1$.

For the transfer task, separate ANOVAs were conducted for the two groups with a different number of practice trials, each 
Table 2 Percentage errors (PE) in the transfer tasks of Experiments 1-4

\begin{tabular}{|c|c|c|c|c|c|}
\hline & Practice stimulus/response & Transfer stimulus/response & Number of practice trials & Compatible & Incompatible \\
\hline \multirow[t]{3}{*}{ Experiment 1} & - & Location/Vocal & - & 2.85 & 1.63 \\
\hline & Location/Vocal & Location/Vocal & 84 & 3.15 & 3.22 \\
\hline & Word/Vocal & Location/Vocal & 84 & 2.06 & 1.89 \\
\hline \multirow[t]{5}{*}{ Experiment 2} & - & Word/Vocal & - & 2.27 & 4.88 \\
\hline & Location/Vocal & Word/Vocal & 84 & 1.79 & 4.32 \\
\hline & Word/Vocal & Word/Vocal & 84 & 2.58 & 3.66 \\
\hline & Location/Vocal & Word/Vocal & 168 & 2.03 & 3.32 \\
\hline & Word/Vocal & Word/Vocal & 168 & 1.84 & 1.93 \\
\hline \multirow[t]{3}{*}{ Experiment 3} & - & Location/Keypress & - & 2.77 & 3.93 \\
\hline & Location/Vocal & Location/Keypress & 84 & 3.21 & 3.48 \\
\hline & Location/Keypress & Location/Vocal & 84 & 2.52 & 2.96 \\
\hline \multirow[t]{3}{*}{ Experiment 4} & - & Word/Keypress & - & 1.31 & 3.84 \\
\hline & Word/Vocal & Word/Keypress & 168 & 1.82 & 3.22 \\
\hline & Word/Keypress & Word/Vocal & 168 & 1.24 & 3.01 \\
\hline
\end{tabular}

of which involved Practice Mode (physical location vs. spatial word vs. control; between-subject) and Compatibility (compatible vs. incompatible; within-subject) as factors. For RT of the groups with 84 practice trials, the only significant effect was that of Compatibility, $F(1,45)=72.14, M S E=646, p<$ $.001, \eta_{\mathrm{p}}{ }^{2}=.616$, yielding a $44-\mathrm{ms}$ Simon effect $(M \mathrm{~s}=611 \mathrm{~ms}$ and $655 \mathrm{~ms}$ for compatible and incompatible trials). The interaction was not significant, $F(2,45)=2.13, M S E=646, p=$ $.131, \eta_{\mathrm{p}}{ }^{2}=.086$, which indicates that the Simon effect was not modulated by the prior incompatible mappings with word or location stimuli (the Simon effect was $44 \mathrm{~ms}$ for control, $57 \mathrm{~ms}$ for location practice, and $31 \mathrm{~ms}$ for word practice). For PE, the main effect of Compatibility was significant, $F(1,45)=27.03, M S E=3.81, p<.001, \eta_{\mathrm{p}}{ }^{2}=.375$, reflecting a Simon effect of $2.07 \%(M \mathrm{~s}=2.21 \%$ and $4.29 \%$ for compatible and incompatible trials). No other effects were significant, $F_{\mathrm{s}}<1.6, p \mathrm{~s}>.2$.
For RT of the groups with 168 practice trials, a main effect of Compatibility was significant, $F(1,45)=62.00, M S E=$ $310, p<.001, \eta_{\mathrm{p}}^{2}=.579$, yielding a 28 -ms Simon effect $(M \mathrm{~s}=579 \mathrm{~ms}$ and $607 \mathrm{~ms}$ for compatible and incompatible trials), and compatibility interacted with Practice Mode, $F(2$, $45)=7.03, M S E=310, p<.002, \eta_{\mathrm{p}}{ }^{2}=.238$. As compared to the Simon effect for the control group $(M=44 \mathrm{~ms})$, the effect was significantly smaller for those who practiced with spatial words $(M=11 \mathrm{~ms} ; t(30)=3.92, p<.001)$ but not for those who practiced with physical locations $(M=30 \mathrm{~ms} ; t(30)=$ $1.35, p=.187)$. The main effect of Practice Mode was not significant, $F(2,45)<1$. For PEs, the main effect of Compatibility was significant, $F(1,45)=8.02, M S E=5.28, p<.007$, $\eta_{\mathrm{p}}{ }^{2}=.151$, yielding the Simon effect $(M \mathrm{~s}=2.05 \%$ and $3.38 \%$ for compatible and incompatible trials). The main effect of Practice Mode approached the .05 level, $F(2,45)=2.73$, $M S E=8.36, p=.076$, reflecting higher error rate for the
Fig. 2 Response time (RT) as a function of Practice Mode (control, location, word) with 84 practice trials and 168 practice trials in Experiment 2; the numbers are the Simon effects and an asterisk indicates a significant reduction of the Simon effect compared to the No Practice condition; error bars are standard errors of the means

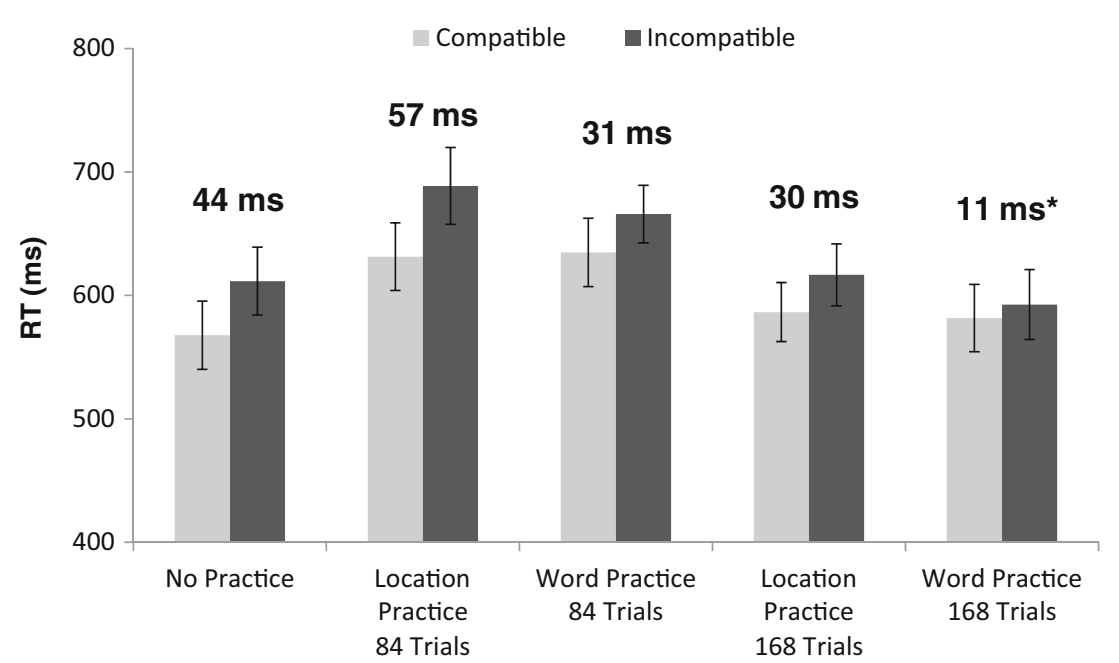


control group $(M=3.58 \%)$ than for the other two $(M \mathrm{~s}=2.68$ $\%$ and $1.89 \%$ for physical location and spatial words, respectively). These two factors did not interact, $F(2,45)=2.43, p>$ .1 .

Including the data from Experiment 1 and the groups with 168 practice trials in Experiment 2, an additional 2 (Practice Mode: physical location vs. spatial word) $\times 2$ (Transfer Mode: physical location vs. spatial word) $\times 2$ (Compatibility) ANOVA was conducted, of which the main focus was the interaction among the three factors. As expected, it revealed a significant 3-way interaction, $F(1,60)=20.66, M S E=257$, $p<.001, \eta_{p}{ }^{2}=.256$, reflecting the outcomes that the Simon effect was reduced when the stimulus type matched between the practice and transfer tasks but not when they mismatched.

\section{Discussion}

Two outcomes of the present experiment are particularly relevant. First, the incompatible spatial mappings were transferred to the Simon task after practice with spatial words for 168 trials but not for 84 trials. Spatial words required more practice trials than physical location stimuli, which produced the transfer effect only after 84 trials in Experiment 1 . The results are consistent with those obtained with manual responses (Proctor et al., 2009). Therefore, the acquisition of spatial associations depends on the type of stimuli, not the degree of set-level compatibility. Second, with longer practice, the incompatible spatial mappings transferred to the Simon task only after practice with spatial words but not after practice with physical locations when stimuli were spatial words in the transfer task. This outcome corroborates the result of Experiment 1 , supporting the conclusion that transfer of new spatial associations depends on contextual overlap between the practice and transfer tasks with respect to the type of stimuli, but set-level compatibility plays no major role.

\section{Experiment 3}

The preceding two experiments varied the type of stimuli used in the practice and transfer tasks, while maintaining the response mode between the tasks. The following two experiments varied the response modes in the practice and transfer tasks while maintaining the type of stimuli. These manipulations allowed dissociating the role of the response mode from that of set-level compatibility. For both manual and vocal response modes, only short practice is required to yield the transfer effect when participants responded to physical location stimuli. Also, for both manual and vocal responses, contextual match between the practice and transfer tasks determined transfer of spatial associations. The similarity between the outcomes with manual and vocal responses raises the possibility that the differences between the two response modes are superficial and a common mechanism underlies both situations (see e.g., Stoet \& Hommel, 1999; Wühr, 2006). Therefore, Experiments 3 and 4 examined whether acquired spatial associations transfer between the two modes of responding.

In the present experiment, physical location stimuli were used for the practice and transfer tasks, and half of the participants responded vocally in the practice task and manually (by pressing left and right keys) in the transfer task, whereas the other half responded manually in the practice task and vocally in the transfer task. As short practice is sufficient to acquire incompatible spatial associations for physical location stimuli with manual and vocal responses, all participants had only 84 practice trials. If a common mechanism underlies the two modes of responding, transfer of incompatible spatial associations would be expected in the Simon task regardless of the response modes used in the two tasks.

\section{Method}

Participants Forty-eight undergraduate students at Purdue University were newly recruited for the present experiment. They received experimental credits for their psychology courses. None had participated in the preceding experiments, and all reported having normal or corrected-to-normal visual acuity and normal color vision.

Apparatus, stimuli, and procedure Stimuli were the location stimuli (white circles in the practice task, and red and green circles in the transfer task) used in Experiment 1. The practice task consisted of 84 trials. The apparatus was the same as that used in Experiment 1. Participants were assigned randomly to one of three groups of 16 participants each. The first group performed the practice task using a keyboard, pressing the " $\mathrm{z}$ " (left) and "/" (right) keys; in the transfer task, this group performed the Simon task by speaking into a microphone. The second group used these response modes in the reversed order: they spoke into a microphone in the practice task and pressed keys in the transfer task. The third group served as the control group for the second group (who performed the Simon task with keypresses); those in the control group only performed the Simon task. For those who performed the transfer task with vocal responses, the no-practice group in Experiment 1 served as the control.

Results

Percentages of discarded trials were $0.30 \%$ for the practice task and $2.32 \%$ for the transfer task. The data were analyzed separately for those who used the keyboard in the transfer task and those who spoke into a microphone. RTs and PEs for the practice task are shown in Table 1. 
Vocal practice to manual Simon For the transfer task, RTs and PEs were submitted to 2 (Practice Mode: keyboard vs. control) $\times 2$ (Compatibility: compatible vs. incompatible) ANOVAs. RT showed a main effect of Compatibility, $F(1$, $30)=10.39, M S E=346, p=.003, \eta_{\mathrm{p}}{ }^{2}=.257$, yielding a 15-ms Simon effect $(M s=568 \mathrm{~ms}$ and $583 \mathrm{~ms}$ for compatible and incompatible trials; see Fig. 3). Although the magnitude of the Simon effect appears small as compared to that obtained with vocal responses (see Experiments 1 and 2), this is a typical size when responses are keypresses (e.g., Proctor et al., 2009; Yamaguchi \& Proctor, 2009). No other effects were significant, $F \mathrm{~S}<1$. PE did not yield any significant effect, $F \mathrm{~s}<1.4, p \mathrm{~s}>.2$ (see Table 2 ).

Manual practice to vocal Simon For the transfer task, RTs and PEs were analyzed in the same manner as for the Keypress Simon group. RT showed a significant main effect of Compatibility, $F(1,30)=30.00, M S E=221, p<.001, \eta_{\mathrm{p}}{ }^{2}=.500$, yielding a $20-\mathrm{ms}$ Simon effect $(M \mathrm{~s}=550 \mathrm{~ms}$ and $571 \mathrm{~ms}$ for compatible and incompatible trials; see Fig. 3). The factor interacted with Practice Mode, $F(1,30)=7.27, M S E=221$, $p=.011, \eta_{\mathrm{p}}{ }^{2}=.195$. The Simon effect was smaller for those who performed the practice task with the keyboard $(M=10$ $\mathrm{ms})$ than for the control group $(M=30 \mathrm{~ms})$. The main effect of Practice Mode was not significant, $F(1,30)<1.4$. PE did not involve any significant effect, $F_{\mathrm{S}}<2.1, p \mathrm{~s}>.1$ (see Table 2).

\section{Discussion}

The present results showed little evidence that prior spatial associations transferred to the Simon task when the response mode was vocal in the practice task and manual in the transfer task. However, transfer of incompatible mappings was observed when the response mode was manual in the practice task and vocal in the transfer task. Although this transfer effect

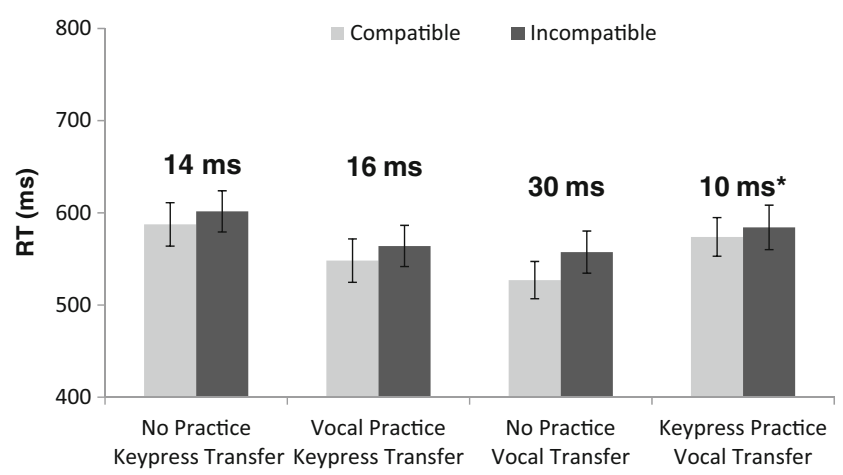

Fig. 3 Response time (RT) as a function of Practice Mode (control vs. keypress or vocal) for the keypress and vocal Simon conditions of Experiment 3; the numbers are the Simon effects and an asterisk indicates a significant reduction of the Simon effect compared to the No Practice condition (the data for No Practice Vocal Transfer are those from the corresponding group of Experiment 1); error bars are standard errors of the means is consistent with the idea that a common mechanism underlies the Simon task with manual and vocal responses, the lack of the transfer effect in the other group complicates the issue. This curious pattern of results may be because responses were represented differently according to the mode of responding in the practice task (e.g., Ansorge \& Wühr, 2009). For instance, participants might have continued using response codes that they adopted in the practice task in order to represent responses in the transfer task when they switched from manual responses to vocal responses, thus representing vocal responses based on spatial codes. Participants might have adopted lexical codes to represent vocal responses in the practice task, but it may have been more difficult to continue using the same lexical codes to represent keypresses in the transfer task because stimuli were physical locations. Although speculative, such asymmetric transfer of response codes from the practice task to the transfer task could explain the present results. If so, it is not always possible to predict transfer of learned associations merely by looking at contextual overlap at the surface features of the tasks. However, it is noteworthy that the 20-ms reduction of the Simon effect in the present experiment was smaller than the 31-ms reduction obtained in Experiment 1 in which the response mode was vocal in both tasks. Thus, although spatial associations transferred to the Simon task, contextual match still appeared to have played a role in the present experiment.

\section{Experiment 4}

Experiment 4 was essentially the same as Experiment 3 but used spatial words in the practice and transfer tasks. The practice task consisted of 168 trials, as Experiment 2 indicated that spatial words required more practice trials to produce the transfer effect. If set-level compatibility played any role in the results of Experiment 3 (e.g., asymmetric transfer effects), the Simon effect with manual responses should be reduced after practice with vocal responses. If contextual match with respect to the response mode is the primary determinant of the transfer effect, practice with the incompatible-mapping task should have little influence on the Simon effect because the mode of responding differed between the practice and transfer tasks.

Method

Participants A new group of 48 participants were recruited from the same subject pool as in the preceding experiments. None had participated in the preceding experiments.

Apparatus, stimuli, and procedure The procedure was the same as that in Experiment 3, but stimuli were spatial words in both practice and transfer tasks. The practice task consisted 
of 168 trials. A group of participants was assigned to the condition in which they responded vocally in the practice task and manually in the transfer task; another group was assigned to the condition in which they responded manually in the practice task and vocally in the transfer task; and the last group was assigned to the condition in which they performed only the transfer task with manual responses. The last group served as the control group for those who used keypresses in the transfer task. The control group in Experiment 2, who only performed the transfer task with vocal responses, served as the control for those who used vocal responses in the transfer task. Experiment 4 was identical with Experiment 3 in other respects.

\section{Results}

Percentages of discarded trials were $1.36 \%$ for the practice task and $1.76 \%$ for the transfer task. As in Experiment 3, the data were analyzed separately for those who used the keyboard in the transfer task and those who spoke into a microphone. RTs and PEs are shown in Table 1.

Vocal practice to manual Simon For the transfer task, RTs and PEs were submitted to 2 (Practice Mode: keyboard vs. control) $\times 2$ (Compatibility: compatible vs. incompatible) ANOVAs. For RT, there was a main effect of Compatibility, $F(1,30)=15.09, M S E=574, p<.001, \eta_{\mathrm{p}}{ }^{2}=.335$, yielding a 23-ms Simon effect $(M s=468 \mathrm{~ms}$ and $491 \mathrm{~ms}$ for compatible and incompatible trials; see Fig. 4), but no other effect was significant, $F \mathrm{~s}<1$. Also, for PE, there was a main effect of Compatibility, $F(1,30)=9.56, M S E=6.46, p=.004, \eta_{\mathrm{p}}{ }^{2}=$ .242 , yielding a $1.96 \%$ Simon effect (see Table 2); no other effect reached significance, $F_{\mathrm{S}}<1$.

Manual practice to vocal Simon RTs and PEs were analyzed in the same manner as for the Keypress Simon group. For RT,

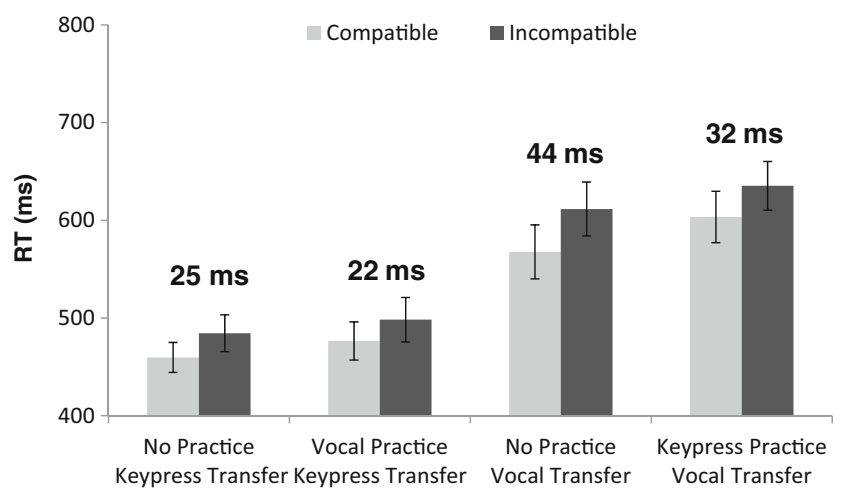

Fig. 4 Response time (RT) as a function of Practice Mode (control vs. keypress or vocal) for the keypress and vocal Simon conditions of Experiment 3; the numbers are the Simon effects (the data for No Practice Vocal Transfer are those from the corresponding group of Experiment 2); error bars are standard errors of the means there was a main effect of Compatibility, $F(1,30)=60.43$, $M S E=379, p<.001, \eta_{\mathrm{p}}{ }^{2}=.668$, yielding a 36-ms Simon effect $(M \mathrm{~s}=587 \mathrm{~ms}$ and $623 \mathrm{~ms}$ for compatible and incompatible trials; see Fig. 4). No other effect was significant, $F_{\mathrm{S}}<$ $1.3, p \mathrm{~s}>$.2. Also, for PE, the main effect of Compatibility was significant, $F(1,30)=16.24, M S E=4.73, p<.001, \eta_{\mathrm{p}}{ }^{2}=$ .351 , reflecting a $2.19 \%$ Simon effect (see Table 2 ). There was also a significant main effect of Practice Mode, $F(1,30)=$ $5.16, M S E=6.52, p=.030, \eta_{\mathrm{p}}{ }^{2}=.147$, reflecting more accurate response for those who performed the practice task $(M=$ $2.12 \%)$ than for the control group $(M=3.58 \%)$. However, there was no significant interaction between the two factors, $F$ $<1$.

\section{Discussion}

In contrast to Experiment 3, the present experiment did not show any reduction of the Simon effect in either group. Again, the outcome indicates the importance of contextual match between the practice and transfer tasks in terms of response mode. If the modulation of the Simon effect in Experiment 3 was due to the degree of set-level compatibility being higher for manual responses (because stimuli were physical locations), the Simon effect should have been modulated after practice with vocal responses that were highly compatible with spatial words. The lack of such outcome corroborates the conclusion that transfer of newly acquired spatial associations depends primarily on contextual match between the practice and transfer tasks, but set-level compatibility plays no major role in transfer. Although this conclusion rests on the null effects of the present experiment, it is consistent with the findings of Experiments 1 and 2, in which there were significant interactions between compatibility and stimulus type, indicating that the match of stimulus type between the practice and transfer tasks is a strong determinant of the transfer effect, but set-level compatibility is not.

\section{General discussion}

The Simon effect has been a useful tool in investigating the fundamental mechanisms underlying response selection (Hommel, 2011; Kornblum et al., 1990; Yamaguchi \& Proctor, 2012). In the present study, the transfer paradigm was used to examine the role of set-level compatibility and response mode in transfer of learning in choice reactions. The Simon effect can be modulated when participants perform an incompatible-mapping task prior to the Simon task (e.g., Proctor \& Lu, 1999; Tagliabue et al. 2000), but little has been known about the role of set-level compatibility in the acquisition of incompatible spatial associations and transfer of these associations to the Simon task. The present study extended the previous findings by dissociating the contributions of stimulus 
type, response mode, and set-level compatibility between them, and also by assessing the contextual match between the practice and transfer tasks using vocal responses. The results of the four experiments are consistent, showing that contextual match with respect to the type of stimuli and the mode of responding is the primary determinant of transfer of incompatible mappings to the Simon task. There was little evidence that set-level compatibility plays a major role in the acquisition or transfer of associations.

The first main finding of the present study is that newly acquired incompatible spatial associations can transfer to the Simon task when responses are made vocally: this is the first demonstration of transfer using vocal responses. A previous study has used the Stroop task after practice with the incompatible-mapping task using manual responses (Marini et al., 2011). The Stroop effect was modulated after the incompatible-mapping task if responses were made manually but not when responses were made vocally. This finding led to the proposal that transfer of incompatible mapping is based on the acquisition of an abstract rule that depends on the spatial distinction between alternative responses (e.g., left vs. right response keys). However, the present finding that the Simon effect can be modulated with vocal responses indicates that it is not due to the mode of responding or the lack of spatial distinction within the vocal response set that prevented the incompatible mapping to transfer to the subsequent task. Instead, transfer of associations is prevented due to the change in the response mode between the practice and transfer tasks.

The second main finding is that the Simon effect with vocal responses was modulated more strongly when both stimuli in the practice and transfer sessions were physical locations (Experiment 1) or spatial words (Experiment 2), as compared to when stimuli were physical locations in one task and spatial words in the other task. These results are consistent with the findings in the previous study using manual responses (Proctor et al., 2009), highlighting the importance of contextual overlap. On the other hand, spatial words were more compatible with vocal responses than were physical location stimuli, but there was little evidence that this higher set-level compatibility plays any role in transfer of spatial associations. There is evidence indicating that similarity between two stimulus events facilitates the association between them (e.g., Yamaguchi \& Proctor, 2009), but the present results clearly showed that such a finding does not generalize to the acquisition of incompatible associations between stimulus and response.

The third main finding is that the ease of acquiring new spatial associations depended on the type of stimuli; in particular, associations are acquired more quickly with physical location stimuli than with spatial words. The importance of stimulus type in the acquisition of spatial associations was also indicated by a previous study with manual responses (Proctor et al., 2009). The present study reinforces such a finding by showing that even with vocal responses, physical locations required fewer practice trials than did spatial words. Tagliabue et al. (2000) suggested that it was an acquisition of temporary $\mathrm{S}-\mathrm{R}$ associations during the practice task that counters preexisting S-R associations representing compatible mappings. This account would suggest a critical role of associability between stimulus and response. Marini et al. (2011) suggested that it may be a general rule of "emit an alternative response" that is acquired during practice. This account would suggest a critical role of response mode in transfer of new associations. However, neither account speaks much to the finding that incompatible mappings transfer more easily with physical locations than spatial words, regardless of the mode of responding. A tentative explanation for this outcome is that spatial information is conveyed more effectively with physical locations than with spatial words, so it is associated with responses better. This possibility needs to be subjected to further investigation.

Finally, the present study also demonstrated the importance of contextual overlap between the practice and transfer tasks with respect to response mode. In Experiments 3 and 4, there was little influence of the prior incompatible-mapping task on the Simon task when response mode differed between the two tasks, despite the fact that stimuli were of the same type between the tasks. Thus, contextual overlap in the mode of responding is also an important determinant of the transfer effect (also see Yamaguchi \& Proctor, 2009). A puzzling finding is the outcome that the Simon effect was modulated in Experiment 3 when responses were made manually in the practice task and vocally in the transfer task. There is a study indicating that response representation adopted in a previous task can transfer to a subsequent task (Ansorge \& Wühr, 2009); thus, it is possible that spatial codes used to represent manual responses in the practice task were also adopted to represent vocal responses in the transfer task. However, the results were not replicated when stimuli were spatial words in Experiment 4. Hence, this aspect of the study requires further scrutiny in future investigations.

A picture that emerges from the present study is that transfer of learned associations depends on contextual match between the practice and transfer tasks with respect to stimulus type and response mode, but not much on the relation between the two factors (set-level compatibility). Also, the results indicate that the acquisition of spatial associations depends primarily on stimulus type: spatial associations can be acquired more easily based on physical locations than on the spatial meanings of words. This makes sense because spatial information is extracted from the actual stimulus locations more quickly than from the concepts of locations, as implied by faster responses to physical location stimuli than to spatial words in the practice task even if responses were made vocally (see Table 1). A question still remains as to what was actually learned during practice, whether it is some abstract rule of 
stimulus-to-response translation or simple link between stimulus and response codes (Baroni et al., 2013). However, it is clear from the present findings that acquisition depends on the manner in which spatial information is represented.

The contextual dependence of transfer of spatial associations is consistent with general findings in the studies of animal and human learning (Bouton, 1993; Godden \& Baddeley, 1975; Healy et al., 2006; Tulving \& Thomson, 1973), and it is well supported by the previous studies using the transfer paradigm (Baroni et al., 2013; Proctor et al., 2009; Marini et al., 2011; Yamaguchi \& Proctor, 2009). Although transfer can occur in the face of some changes in task contexts, such as sensory modality (Tagliabue et al., 2002) and type of manual responses (Yamaguchi \& Proctor, 2009), especially after extensive training (Proctor et al., 2007; Vu, 2007), all of these cases are situations in which different stimuli and responses are used to convey physical locations; thus, all stimuli and responses are likely represented by the same spatial codes. Hence, it would be interesting to see whether transfer of spatial associations can be induced between different stimulus types (e.g., words vs. locations) and response mode (e.g., keypress vs. vocal) after more extensive training. If more extensive training allows transfer between different stimulus types or between response modes, then it would be important to determine whether any qualitative changes emerge in the way these different stimuli and responses are represented cognitively. Such studies would provide important implications as to how transfer of learning can be promoted in more practical task settings, such as in educational or occupational settings.

Acknowledgments We thank Alice Healy, Iring Koch, and Joseph Magliano, for insightful comments on an earlier draft of the paper.

\section{References}

Ansorge, U., \& Wühr, P. (2009). Transfer of response codes from choice responses to go-nogo tasks. Quarterly Journal of Experimental Psychology, 62, 1216-1235. doi:10.1080/17470210802426866

Baldo, J. V., Shimamura, A. P., \& Prinzmetal, W. (1998). Mapping symbols to response modalities: Interference effects on Stroop-like tasks. Perception \& Psychophysics, 60, 427-437. doi:10.3758/ BF03206864

Baroni, G., Yamaguchi, M., Chen, J., \& Proctor, R. W. (2013). Mechanisms underlying transfer of task-defined rules across feature dimensions. Experimental Psychology, 60, 410-424. doi:10.1027/ 1618-3169/a000214

Bouton, M. E. (1993). Context, time, and memory retrieval in the interference paradigms of Pavlovian learning. Psychological Bulletin, 114, 80-99. doi:10.1037/0033-2909.114.1.80

Fitts, P. M., \& Seeger, C. (1953). S-R compatibility: Spatial characteristics of stimulus and response codes. Journal of Experimental Psychology, 46, 199-210. doi:10.1037/h0062827

Godden, B. R., \& Baddeley, A. D. (1975). Context-dependent memory in two natural environments: On land and underwater. British Journal of Psychology, 66, 325-331. doi:10.1111/j.2044-8295.1975. tb01468.x
Hazeltine, E., Ruthruff, E., \& Remington, R. W. (2006). The role of input and output modality pairings in dual-task performance: Evidence for content-dependent central interference. Cognitive Psychology, 52, 291-345. doi:10.1016/j.cogpsych.2005.11.001

Healy, A. F., Wohldmann, E. L., Sutton, E. M., \& Bourne, L. E., Jr. (2006). Specificity effects in training and transfer of speeded responses. Journal of Experimental Psychology: Learning, Memory, and Cognition, 32, 534-546. doi:10.1037/0278-7393. 32.3.534

Hommel, B. (2011). The Simon effect as tool and heuristic. Acta Psychologica, 136, 189-202. doi:10.1016/j.actpsy.2010.04.011

Kornblum, S., Hasbroucq, T., \& Osman, A. (1990). Dimensional overlap: Cognitive basis for stimulus-response compatibility - A model and taxonomy. Psychological Review, 97, 253-270. doi:10.1037/0033295X.97.2.253

Kornblum, S., \& Lee, J.-W. (1995). Stimulus-response compatibility with relevant and irrelevant stimulus dimensions that do and do not overlap with the response. Journal of Experimental Psychology: Human Perception and Performance, 21, 855-875. doi:10.1037/00961523.21.4.855

Lu, C.-H., \& Proctor, R. W. (1994). Processing of an irrelevant location dimension as a function of the relevant stimulus dimension. Journal of Experimental Psychology: Human Perception and Performance, 20, 286-298. doi:10.1037/0096-1523.20.2.286

Lu, C.-H., \& Proctor, R. W. (1995). The influence of irrelevant location information on performance: A review of the Simon and spatial Stroop effects. Psychonomic Bulletin \& Review, 2, 174-207. doi: 10.3758/BF03210959

Marini, M., Iani, C., Nicoletti, R., \& Rubichi, S. (2011). Between-task transfer of learning from spatial compatibility to a color Stroop task. Experimental Psychology, 58, 473-479. doi:10.1027/1618-3169/ a000115

Proctor, R. W., \& Lu, C.-H. (1999). Processing irrelevant location information: Practice and transfer effects in choice-reaction tasks. Memory \& Cognition, 27, 63-77. doi:10.3758/BF03201214

Proctor, R. W., \& Vu, K.-P. L. (2006). Stimulus-response compatibility principles: Data, theory, and application. Boca Raton, FL: CRC Press.

Proctor, R. W., \& Wang, H. (1997). Set- and element-level stimulusresponse compatibility effects for different manual response sets. Journal of Motor Behavior, 29, 351-365. doi:10.1080/ 00222899709600021

Proctor, R. W., Yamaguchi, M., Zhang, Y., \& Vu, K. P.-L. (2009). Influence of visual stimulus mode on transfer of acquired spatial associations. Journal of Experimental Psychology: Learning, Memory, and Cognition, 35, 434-445. doi:10.1037/a0014529

Proctor, R. W., Yamaguchi, M., \& Vu, K. P.-L. (2007). Transfer of noncorresponding spatial associations to the auditory Simon task. Journal of Experimental Psychology: Learning, Memory, and Cognition, 33, 245-253. doi:10.1037/0278-7393.33.1.245

Stephan, D. N., \& Koch, I. (2010). Central cross-talk in task switching: Evidence from manipulating input-output modality compatibility. Journal of Experimental Psychology: Learning, Memory \& Cognition, 36, 1075-1081. doi:10.1037/a0019695

Stoet, G., \& Hommel, B. (1999). Action planning and the temporal binding of response codes. Journal of Experimental Psychology: Human Perception and Performance, 28, 1345-1363. doi:10.1037/00961523.25.6.1625

Tagliabue, M., Zorzi, M., Umiltà, C., \& Bassignani, F. (2000). The role of long-term-memory links and short-term-memory links in the Simon effect. Journal of Experimental Psychology: Human Perception and Performance, 26, 648-670. doi:10.1037/0096-1523.26.2.648

Tagliabue, M., Zorzi, M., \& Umiltà, C. (2002). Cross-modal re-mapping influences the Simon effect. Memory \& Cognition, 30, 18-23. doi: 10.3758/BF03195261 
Tulving, E., \& Thomson, D. M. (1973). Encoding specificity and retrieval processes in episodic memory. Psychological Review, 80, 352-373. doi: $10.1037 / \mathrm{h} 0020071$

$\mathrm{Vu}, \mathrm{K}$.-P. L. (2007). Influences on the Simon effect of prior practice with spatially incompatible mappings: Transfer within and between horizontal and vertical dimensions. Memory \& Cognition, 35, 14631471. doi:10.3758/BF03193616

Vu, K.-P. L., Proctor, R. W., \& Urcuioli, P. (2003). Transfer effects of incompatible location-relevant mappings on a subsequent visual or auditory Simon task. Memory \& Cognition, 31, 1146-1152. doi:10. 3758/BF03196135

Wang, H., \& Proctor, R. W. (1996). Stimulus-response compatibility as a function of stimulus code and response modality. Journal of Experimental Psychology: Human Perception and
Performance, 22, 1201-1217. doi:10.1037/0096-1523.22.5. 1201

Welford, A. T. (1960). The measurement of sensory-motor performance: Survey and reappraisal of twelve years' progress. Ergonomics, 3, 189-229. doi:10.1080/00140136008930484

Wühr, P. (2006). The Simon effect in vocal responses. Acta Psychology, 121, 210-226. doi:10.1016/j.actpsy.2004.12.003

Yamaguchi, M., \& Proctor, R. W. (2009). Transfer of learning in choicereactions: Contributions of specific and general components of manual responses. Acta Psychologica, 130, 1-10. doi:10.1016/j.actpsy. 2008.09.008

Yamaguchi, M., \& Proctor, R. W. (2012). Multidimensional vector model of stimulus-response compatibility. Psychological Review, 119, 272-303. doi:10.1037/a0026620 